\title{
On the Average Number of $\boldsymbol{k}$-Sets
}

\author{
Paul H. Edelman* \\ School of Mathematics, University of Minnesota, Minneapolis, MN 55455, USA
}

\begin{abstract}
In this paper we compute in closed form the average number of $k$-sets of an allowable sequence.
\end{abstract}

\section{Introduction and Notation}

Because of their applications to computational geometry there has recently been a spurt of interest in the properties of the number of $k$-sets of a configuration of $n$ points in the plane. One common approach to this study is to generalize the questions to the $k$-sets of an allowable circular sequence altering the problem from one of geometry to one of combinatorics. The purpose of this paper is to compute explicitly the average number of $k$-sets in an allowable sequence.

We begin by fixing some notation and terminology. Fix $n>1$ and let $S_{n}$ be the set of permutations of the set $[n]=\{1,2, \ldots, n\}$. An allowable circular sequence of permutations is a sequence of permutations in $S_{n}$

$$
P=\left(P_{0}, P_{1}, \ldots, P_{2 L}=P_{0}\right)
$$

where $L=\left(\begin{array}{l}n \\ 2\end{array}\right)$ and the permutations satisfy the conditions:

(1) $P_{i}$ and $P_{i+L}$ are in reverse order (addition is taken modulo $2 L$ ); and

(2) $P_{k+1}$ differs from $P_{k}$ by an adjacent transposition.

Two allowable circular sequences $P$ and $Q$ are considered the same if there is a $c \geq 0$ such that $P_{i}=Q_{i+c}$ for all $0 \leq i \leq 2 L$. A $k$-set of an allowable circular sequence $P$ is an initial segment of size $k$ of one of the $P_{i}$.

\footnotetext{
* This work supported by a grant from the NSF.
} 
Without loss of generality let us suppose that all of our allowable circular sequences have the property that $P_{0}=12 \ldots n$. For each allowable circular sequence $P$ construct an $L$-tuple of adjacent transpositions in $S_{n} \tau=\left(\tau_{1}, \tau_{2}, \ldots, \tau_{L}\right)$ in the letters where $\tau_{j}=(i+1)$ if and only if $P_{j-1}$ differs from $P_{j}$ by a transposition in the $i$ th position. It is easy to see that $\tau$ has the property that

$$
\prod_{i=1}^{L} \tau_{i}=n n-1 \ldots 1=\hat{1}
$$

where the product is taken in $S_{n}$. The sequence $\tau$ is called a reduced expression of $\hat{1}$, i.e., a minimal length factorization of $\hat{1}$ into adjacent transpositions. Moreover, the image under this map of the allowable circular sequences is exactly the set of reduced expressions of $\hat{1}$. Finally, let $\mathscr{R}(n)$ be the set of words of length $L$ in the letters $\{1,2, \ldots, n-1\}$ representing the set of all reduced expressions of $\hat{1}$ by the map

$$
\left(\tau_{1}, \tau_{2}, \ldots, \tau_{\Sigma}\right) \rightarrow \omega_{1} \omega_{2} \cdots \omega_{L},
$$

where $\omega_{i}=j$ if and only if $\tau_{i}=(j j+1)$.

The key observation here (see [EW] and [AG]) is that the number of $k$-sets of an allowable sequence is equal to the number of occurrences of $k$ and $n-k$ in the word representing the related reduced expression. If $n$ is even then the number of $n / 2$-sets is twice the number of occurrences of $n / 2$.

Let $C(n)=|\mathscr{R}(n)|$. In [EG] a bijection was presented showing that $C(n)$ was equal to the number of Young tableaux of shape $\lambda^{[n]}=n-1, n-2, \ldots, 1$, a result previously obtained by Stanley [St]. The number of such tableaux can be explicitly computed thanks to the hook formula of Frame et al. [FRT] (see also [Kn, p. 63]). In the next section we will need this bijection. Also in the next section, we will be concerned with the partitions $\lambda_{k}^{[n]}$ which are partitions of $\left(\begin{array}{l}n \\ 2\end{array}\right)-1$ obtained from $\lambda^{[n]}$ by removing the corner cell $(n-k, k)$. The number of Young tableaux $\lambda_{k}^{[n]}$ is also easily computed using the hook formula.

\section{Theorems}

In this section we prove the main result of the paper, i.e., a closed-form expression for the average number of $k$-sets of an allowable sequence. Recall that $\mathscr{R}(n)$ is the set of all words which represent reduced expressions of $\hat{1}$ in $S_{n}$. The reader can check the following easy computation.

Lemma 2.1. For all $n>1$ and $1 \leq i \leq n-1$ we have

$$
(i+1) \hat{1}=\hat{1}(n-i n-i+1) \text {. }
$$

Lemma 2.2. If $n>1$ and $\omega_{1} \omega_{2} \cdots \omega_{L} \in \mathscr{R}(n)$, then $\omega_{2} \omega_{3} \cdots \omega_{L}\left(n-\omega_{1}\right) \in \mathscr{R}(n)$. 
Proof. This follows directly from Lemma 2.1 and the definition of $\mathscr{R}(n)$.

Theorem 2.3. For all $1 \leq i \leq n-1$ and $1 \leq k \leq L$ the number of words

$$
\omega=\omega_{1} \omega_{2} \cdots \omega_{L} \in \mathscr{R}(n)
$$

where $\omega_{k}=i$ is independent of $k$.

Proof. Let $r(i, k)$ be the number of words described above. We will show that $r(i, k)=r(i, k-1)$ for all $2 \leq k \leq L$. The theorem then follows by induction.

Let $\omega=\omega_{1} \omega_{2} \cdots \omega_{L}$ be a word counted by $r(i, k)$. It then follows from Lemma 2.2 that $\omega^{\prime}=\omega_{2} \omega_{3} \cdots\left(n-\omega_{1}\right)$ is a word counted by $r(i, k-1)$ and that this map is a bijection between the two sets.

Corollary 2.4. For $n>1$ and $1 \leq k \leq n-1$, the total number of occurrences $k$ among all words in $\mathscr{R}(n)$ is

$$
\left(\begin{array}{l}
n \\
2
\end{array}\right) D(n)_{k}
$$

where $D(n)_{k}$ is the number of words in $\mathscr{R}(n)$ whose last letter is $k$.

In order to evaluate $D(n)_{k}$ we will use the theory developed in [EG] to count $C(n)$. In [EG, Theorem 5.4] a bijection is given between Young tableaux of staircase shape and the words in $\mathscr{R}(n)$. A cursory look at this bijection will allow the reader to conclude that

Lemma 2.5. For $1 \leq k \leq n-1$, the number of words in $\mathscr{R}(n)$, whose last letter is $k$, is equal to the number of Young tableaux of staircase shape with the number $L$ in position $(n-k, k)$. Hence $D(n)_{k}$ equals the number of Young tableaux of shape $\lambda_{k}^{[n]}$.

We can now apply the hook formula to compute $D(n)_{k}$ explicitly. For our future use it will be useful to express this number in terms of $C(n)$. Notice that the only difference in the hook lengths of the common cells of $\lambda^{[n]}$ and $\lambda_{k}^{[n]}$ is that, in the $k$ th column and the $n-k$ th row of $\lambda_{k}^{[n]}$, the hook lengths have decreased by one from those of $\lambda^{[n]}$. We thus conclude that

Lemma 2.6. For $n>1$ and $1 \leq k \leq n-1$, we have

$$
D(n)_{k}=\frac{1}{\left(\begin{array}{l}
n \\
2
\end{array}\right)} C(n) \frac{13 \cdots(2 k-1)}{24 \cdots(2 k-2)} \frac{13 \cdots(2(n-k)-1)}{24 \cdots(2(n-k)-2)}
$$

Combining Corollary 2.4 and Lemma 2.6 (and performing some algebraic manipulations) we arrive at the main theorem. 
Theorem 2.7. For $n>1$ and $1 \leq k \leq n-1$, the average number of occurrences of the letter $k$ among all words in $\mathscr{R}(n)$ is

$$
\frac{(2 k-1)(2(n-k)-1)}{2^{2 n-4}}\left(\begin{array}{c}
2 k-2 \\
k-1
\end{array}\right)\left(\begin{array}{c}
2(n-k-1) \\
n-k-1
\end{array}\right)
$$

It may as yet not be clear that we can extend this result to the average number of $k$-sets. To find the average number of $k$-sets we need to know the total number of occurrences of the letters $k$ and $n-k$. It is, however, easy to see that the map that sends the letter $k$ to $n-k$ extended to all of $\mathscr{R}(n)$ is an involution and hence the total number of occurrences of $k$ and $n-k$ is just twice that of $k$ alone. We thus conclude that

Theorem 2.8. The average number of $k$-sets of an allowable sequence is

$$
\frac{(2 k-1)(2(n-k)-1)}{2^{2 n-5}}\left(\begin{array}{c}
2 k-2 \\
k-1
\end{array}\right)\left(\begin{array}{c}
2(n-k-1) \\
n-k-1
\end{array}\right) .
$$

\section{Final Remarks}

The reader may wonder how the above results relate to the known results about the maximum number of $k$-sets of a configuration of $n$ points in the plane. As an illustration we consider the important (and difficult) case in which $n$ is even and $k=n / 2$. It is known that the number of $k$-sets of a $n$-point configuration in this instance is bounded below by $c n \log (2 n / 3)$ and above by $c(2 n)^{3 / 2}$ [ELSS]. An elementary application of Stirling's formula to Theorem 2.8 shows that in this circumstance the average number of $k$-sets is $(4 / \pi) n+O(1)$. So our result does not lend much insight to the geometric question.

Finally, we might want to generalize this analysis to nonsimple allowable sequences, i.e., allowing reversal of strings instead of only pairs. Unfortunately, the number of such strings is unknown and there seems no easy way to generate them.

\section{References}

[AG] N. Alon and E. Györi. The number of small semispaces of a finite set of points in the plane, J. Combin. Theory Ser. A, 41 (1986), 154-157.

[EG] P. H. Edelman and C. Greene, Balanced tableaux, Adv. in Math., 63 (1987), 42-99.

[ELSS] P. Erdös, L. Lovász, A. Simmons, and E. G. Strauss, Dissection graphs of planar point sets, in $A$ Survey of Combinatorial Theory (J. N. Srivastava et al., eds.), North-Holland, Amsterdam, 1973, pp. 139-149.

[EW] H. Edelsbrunner and E. Welzl, On the number of line separations of a finite set in the plane, J. Combin. Theory Ser. A, 38 (1985), 15-29. 
[FRT] J. S. Frame, G. De B. Robinson, and R. M. Thrall, The hook graphs of $S_{n}$, Canad. J. Math, $6(1954), 316-324$.

[Kn] D. E. Knuth, The Art of Computer Programming, volume 3, Addison-Wesley, Reading, Mass., 1973.

[St] R. P. Stanley, On the number of reduced decompositions of elements of Coxeter groups, European, J. Combin., 5 (1984), 359-372.

Received September 11, 1990, and in revised form June 13, 1991. 